\title{
The carboxylic acid transporters Jen 1 and Jen 2 affect the architecture and fluconazole susceptibility of Candida albicans biofilm in the presence of lactate
}

\author{
Rosana Alves ${ }^{a}$, Sandra Mota a,b, Sónia Silvac, Célia F. Rodriguesc, Alistair J. P. Brown ${ }^{d}$, Mariana Henriquesc, \\ Margarida Casal ${ }^{\mathrm{a}}$ and Sandra Paiva ${ }^{\mathrm{a}}$ \\ ${ }^{a}$ Centre of Molecular and Environmental Biology, Department of Biology, University of Minho, Braga, Portugal; ${ }^{b}$ Centre of Health and \\ Environmental Research, School of Allied Health Sciences, Polytechnic Institute of Porto, Porto, Portugal; 'Centre of Biological Engineering, \\ LIBRO-Laboratório de Investigação em Biofilmes Rosário Oliveira, University of Minho, Braga, Portugal; dMRC Centre for Medical Mycology, \\ Aberdeen Fungal Group, Institute of Medical Sciences, University of Aberdeen, Aberdeen, UK
}

ABSTRACT

Candida albicans has the ability to adapt to different host niches, often glucose-limited but rich in alternative carbon sources. In these glucose-poor microenvironments, this pathogen expresses JEN1 and JEN2 genes, encoding carboxylate transporters, which are important in the early stages of infection. This work investigated how host microenvironments, in particular acidic containing lactic acid, affect C. albicans biofilm formation and antifungal drug resistance. Multiple components of the extracellular matrix were also analysed, including their impact on antifungal drug resistance, and the involvement of both Jen 1 and Jen 2 in this process. The results show that growth on lactate affects biofilm formation, morphology and susceptibility to fluconazole and that both Jen 1 and Jen2 might play a role in these processes. These results support the view that the adaptation of Candida cells to the carbon source present in the host niches affects their pathogenicity.
ARTICLE HISTORY

Received 4 August 2017

Accepted 10 October 2017

\section{KEYWORDS}

Candida albicans; biofilm formation; antifungal drug resistance; alternative carbon sources; lactate; fluconazole

\section{Introduction}

The human fungal pathogen Candida albicans is the main etiological agent of candidiasis and one of the most frequent causes of hospital-acquired infections (Pfaller and Diekema 2007; Pappas et al. 2009). This opportunistic fungus is commonly found as a commensal in the human microbial flora of healthy people. However, in individuals with a weakened immune system, it can overgrow and cause serious or fatal infections. The pathogenicity of $C$. albicans and the high mortality rates associated with these infections are, in part, due to the ability to form biofilms and, consequently, resist the common classes of antifungals (Ramage et al. 2006, 2009). Nevertheless, some fitness attributes, such as the flexibility to utilize a wide range of nutrients, also play an important role in virulence (Brown et al. 2014; Miramón and Lorenz 2017).

Inside the human host, C. albicans faces different $\mathrm{pH}$ environments, from acidic to mildly basic, and the ability to adjust to these fluctuations is essential for its pathogenicity (De Bernardis et al. 1998; Davis 2009; Vylkova et al. 2011; Vylkova and Lorenz 2014). In some glucose-poor niches, such as in the colon and in the vagina, this fungus has also to adapt to changes in the availability of carbon sources, assimilating alternative nutrients, such as lactate or acetate (Staib et al. 1999; Barelle et al. 2006; Ene et al. 2013; Brown et al. 2014). This adaptation requires a metabolic switch (Lorenz and Fink 2001; Lorenz et al. 2004; Vieira et al. 2010; Ene et al. 2013), as verified, for instance, upon phagocytosis (Miramón et al. 2012). Microarray data of $C$. albicans cells internalized by macrophages showed the upregulation of JEN1 (Lorenz et al. 2004), encoding a lactate permease (Soares-Silva et al. 2004), and its close homolog JEN2, encoding a malate and a succinate permease (Vieira et al. 2010). Both Jen1-GFP and Jen2GFP were expressed in macrophages and neutrophils, which are rich in alternative carbon sources, but not in the bloodstream where glucose is abundant (Vieira et al. 2010). These results suggest that lactate in the phagosome might help to sustain C. albicans following phagocytosis.

In addition to the phagosome, lactate is present in ingested foods and in the human body. It is produced at high rates by red blood cells, brain, and muscle, it is present in the urogenital tract and represents almost $2 \%$ of all carbon metabolites originating from the gut microbiota (Flint et al. 2012). This carbon source is also a component 
of the lactated Ringer's solutions, Hartmann's solutions, commonly used intravenously after trauma, surgery or burn injury and whose usage increases the risk of systemic candidiasis and the formation of biofilms in catheters (Pfaller and Diekema 2010), representing a severe problem in modern medicine (Donelli and Vuotto 2014). Growth of C. albicans on physiologically relevant concentrations of lactate affects stress adaptation, antifungal drug resistance, the architecture and proteome of the cell wall, immune detection and, in consequence, the virulence of this fungus (Ene et al. 2012, 2013; Ballou et al. 2016). Lactate-grown cells of $C$. albicans are more resistant to amphotericin $B$ and caspofungin, but more sensitive to miconazole (Ene et al. 2012). They also exhibit distinct compositions in the cell wall proteome and secretome in comparison with glucose-grown cells (Ene et al. 2012). These changes lead to a stronger adherence to plastic surfaces and an increase in biofilm formation on both silicone and plastic surfaces in minimal medium, a condition that generally does not promote the yeast-hyphae transition or classical biofilm formation (Ene et al. 2012).

In this work, host microenvironments, in particular acidic niches that contain lactic acid, were studied regarding their effect on $C$. albicans biofilm formation and susceptibility to the most commonly used antifungal, fluconazole. Little is known about the effect of alternative carbon sources in C. albicans biofilm formation and development as the majority of studies are performed using glucose, as sole carbon source. Here, C. albicans cells were grown in RPMI medium containing different substrates, such as glucose and lactate, and then they were characterized with respect to their ability to form biofilms and resist fluconazole. Multiple components of the extracellular matrix were also analysed, including their impact on drug resistance, and the involvement of both carboxylic acid transporters, Jen 1 and Jen2, on these processes. By clarifying the effect of local nutrients on biofilm formation and antifungal resistance in C. albicans, new and effective treatment strategies can be developed for both mucosal and systemic infections.

\section{Material and methods}

\section{Yeast strains and growth conditions}

Experiments were performed with C. albicans RM1000 (ura3::imm434/ura3::imm434, his1::hisG/his1::hisG) (Negredo et al. 1997) and the double jen1jen2 mutant (ura3::imm434/ura3::imm434 his1::hisG/his1::hisG jen 1:: HIS1/jen1::ura3-, jen2::ura3-/jen2::URA3) (Vieira et al. 2010). C. albicans RM1000 (isogenic to the SC5314 strain) was routinely cultured on YPD (1\% yeast extract, $1 \%$ peptone, $2 \%$ glucose and $2 \%$ agar) plates stored at room temperature. The C. albicans jen 1jen2 mutant strain was maintained on $\mathrm{YNB}$ (yeast nitrogen base $0.67 \% \mathrm{w} \mathrm{v}^{-1}$ ) agar plates supplemented with the appropriate requirements for prototrophic growth. Cells were inoculated in YPD broth and incubated for $16-18 \mathrm{~h}$ at $37^{\circ} \mathrm{C}$ under agitation. After incubation, the cells were harvested by centrifugation at $3,000 \mathrm{~g}$ for $10 \mathrm{~min}$ at $4^{\circ} \mathrm{C}$ and washed twice with phosphate buffered saline (PBS). Pellets were then suspended in PBS and the cellular density adjusted to $1 \times 10^{5} \mathrm{cells} \mathrm{m}^{-1}$ using a Neubauer counting chamber. Biofilm growth was performed using Roswell Park Memorial Institute (RPMI) 1640 medium (Sigma, St Louis, MO, USA) with or without lactic acid $\left(0.5 \%, \mathrm{v} \mathrm{v}^{-1}\right)$. The $\mathrm{pH}$ for the RPMI medium was always set either to 5 with $\mathrm{HCl}$ or to 7 with $\mathrm{NaOH}$.

\section{Minimal inhibitory concentration}

The minimal inhibitory concentration (MIC) assays were performed according to the Clinical and Laboratory Standards Institute M27-A3 document (CLSI 2008) with some modifications, using RPMI 1640 broth supplemented with $0.165 \mathrm{M}$ of MOPS at $\mathrm{pH}$ and with or without adding $0.5 \%$ lactic acid at $\mathrm{pH} 5$. Different concentrations of fluconazole were used, ranging from 0 to $1,250 \mu \mathrm{g} \mathrm{ml}^{-1}$ (Mota et al. 2015). Briefly, a colony of the strain grown in YPD solid medium was resuspended in $5 \mathrm{ml}$ of saline solution $\left(\mathrm{NaCl} 0.85 \%, \mathrm{w} \mathrm{v}^{-1}\right)$ until a cellular density equivalent to $0.5 \mathrm{McF}$ arland standard. The yeast suspensions were diluted $(1: 100)$ in saline solution and diluted again (1:20) in RPMI 1640. This suspension in RPMI 1640 was added to the respective well of microtitre plates containing the specific concentration of fluconazole solutions. Controls without antifungal agents were also performed. The microtitre plates were incubated at $37^{\circ} \mathrm{C}$ for $48 \mathrm{~h}$ under aerobic conditions. The MICs of the antifungal agent against each Candida strain were determined visually and by total number of colony forming units (CFUs). For this purpose, cells corresponding to each condition were serial diluted in PBS and $10 \mu \mathrm{l}$ of each one were plated in YPD. Experiments were performed in triplicate, using three independent biological samples.

\section{Biofilm formation}

Biofilm formation was performed as described by Mota et al. (2015). Briefly, $200 \mu \mathrm{l}$ of $1 \times 10^{5}$ cells ml $^{-1}$ suspensions in the required medium were placed into 96-well polystyrene microtitre plates (Orange Scientific, Brainel'Alleud, Belgium) and incubated at $37^{\circ} \mathrm{C}$ under aerobic conditions with gentle agitation. At $24 \mathrm{~h}$, the entire volume of medium was removed and $200 \mu \mathrm{l}$ of fresh medium were added to each well. In order to study the effect of fluconazole on biofilm formation, different concentrations (50, 
$150,312.5$ and $1,250 \mu \mathrm{g} \mathrm{ml}^{-1}$ ) were prepared in RPMI 1640 medium and added to the $24 \mathrm{~h}$ formed biofilm. The microtitre plates were then incubated for an additional $24 \mathrm{~h}$, totalling $48 \mathrm{~h}$ of biofilm growth.

\section{Biofilm biomass quantification}

After biofilm formation for $48 \mathrm{~h}$, the entire volume of medium was aspirated and non-adherent cells removed by washing once with PBS. Biofilm forming ability was assessed through quantification of the total biomass by crystal violet (CV) staining (Stepanovic et al. 2000). Thus, after washing, biofilms were fixed with $200 \mu \mathrm{l}$ of methanol, which were removed after contact for $15 \mathrm{~min}$. The microtitre plates were allowed to dry at room temperature, and $200 \mu \mathrm{l}$ of CV $\left(1 \%, \mathrm{v} \mathrm{v}^{-1}\right)$ were added to each well and incubated for $5 \mathrm{~min}$. The wells were then gently washed twice with water and $200 \mu \mathrm{l}$ of acetic acid $\left(33 \%, \mathrm{v} \mathrm{v}^{-1}\right)$ were added to release and dissolve the stain. The absorbance of the solution obtained was read in a microtitre plate reader (Bio-Tek Synergy HT, Izasa, Portugal) at $570 \mathrm{~nm}$. The results were presented as absorbance per unit area, Abs $(570 \mathrm{~nm}) \mathrm{cm}^{-2}$. Experiments were performed in triplicate, using three independent biological samples.

\section{Biofilm viability quantification}

The number of cultivable cells in biofilms was determined by the enumeration of CFUs. For that, after biofilm growth for $48 \mathrm{~h}$ and the PBS washing step described previously, the biofilms were scraped from wells in to $200 \mu \mathrm{l}$ PBS and the suspensions were vigorously vortexed to disaggregate cells from the matrix (Silva et al. 2009). Serial 10 -fold dilutions in PBS were plated onto YPD plates and incubated for $24 \mathrm{~h}$ at $37^{\circ} \mathrm{C}$. Complete removal of the biofilm was confirmed by subsequent $\mathrm{CV}$ staining and spectrophotometric reading for inspection of the wells. The results were presented as the total of CFUs per unit area $\left(\log _{10} \mathrm{CFU} \mathrm{cm} \mathrm{cm}^{-2}\right)$. Experiments were performed in duplicate, using three independent biological samples.

\section{Biofilm structure analysis}

Biofilm structure was assessed by scanning electron microscopy (SEM). Biofilms were formed in 24-well polystyrene microtitre plates (Orange Scientific) with $1 \mathrm{ml}$ of $1 \times 10^{5}$ cells ml ${ }^{-1}$ suspensions, as described previously. After $48 \mathrm{~h}$ incubation the biofilms formed were washed with PBS, dehydrated with alcohol (using 70\% ethanol for $10 \mathrm{~min}, 90 \%$ ethanol for $10 \mathrm{~min}$ and $100 \%$ ethanol for $20 \mathrm{~min}$ ) and air-dried. Prior to observation, the base of the wells was mounted onto aluminium stubs, sputter coated with a thin film (15 nm) of Au-Pd (80-20 wt \%) and observed with an ultra-high resolution Field Emission Gun Scanning Electron Microscopy (FEG-SEM; Nova NanoSem 200, FEI Company, OR, USA).

\section{Biofilm matrix extraction}

Biofilms were formed in a 24-well polystyrene microtitre plate (Orange Scientific), for each condition, as described previously. After $48 \mathrm{~h}$, the formed biofilm was washed with PBS, scraped from the wells and resuspended in $5 \mathrm{ml}$ of PBS. The extracted matrices were sonicated (Ultrasonic Processor, Cole-Parmer, IL, USA) for $30 \mathrm{~s}$ at $30 \%$ amplitude and vortexed for $30 \mathrm{~s}$. One $\mathrm{ml}$ of the suspension was used for dry biofilm weight determination and the rest was centrifuged at $5,000 \mathrm{rpm}$ for $5 \mathrm{~min}$ at $4^{\circ} \mathrm{C}$. The supernatant was filtered through a $0.45 \mu \mathrm{m}$ nitrocellulose filter and stored at $-20^{\circ} \mathrm{C}$ until further analysis. The experiments were performed in triplicate, using two independent biological samples.

\section{Protein determination in the biofilm matrix}

The protein quantification was measured using the BCA Protein Assay Kit (Thermo Scientific, Waltham, MA, USA), using bovine serum albumin as standard and following the manufacturer's instructions. Briefly, $25 \mu \mathrm{l}$ of each sample were mixed with $200 \mu$ of BCA Working Reagent. After incubation for $30 \mathrm{~min}$ at $37^{\circ} \mathrm{C}$, the absorbance of the solution obtained was read in a microtitre plate reader (Bio-Tek Synergy HT, Izasa, Portugal) at $562 \mathrm{~nm}$, using PBS as a blank. The values were normalized per $g$ of dry weight of biofilm and presented as $\mathrm{mg}$ of protein per $\mathrm{g}$ of dry weight of biofilm ( $\mathrm{mg} \mathrm{g}^{-1}$ biofilm).

\section{Carbohydrate determination in the biofilm matrix}

Carbohydrate quantification was assessed by the phenol-sulphuric acid method (DuBois et al. 1956), using glucose as standard. Briefly, $500 \mu \mathrm{l}$ of each sample were mixed with $500 \mu \mathrm{l}$ of phenol $\left(50 \mathrm{~g} \mathrm{l}^{-1}\right)$ and 2,500 $\mu \mathrm{l}$ of sulphuric acid (95-95\%). After incubation for $15 \mathrm{~min}$ at room temperature, all polysaccharides and their derivatives were stained orange-yellow and the absorbance of the solution obtained read in a microtitre plate reader (BioTek Synergy HT, Izasa, Portugal) at $490 \mathrm{~nm}$, using PBS as a blank. The values were normalized per $\mathrm{g}$ of dry weight of biofilm and presented as mg of carbohydrate per $\mathrm{g}$ of dry weight of biofilm ( $\mathrm{mg} \mathrm{g}^{-1}$ biofilm).

\section{$\beta-1,3$ Glucan determination in the biofilm matrix}

The matrix $\beta$-1,3-glucan content was determined using the Glucatell $(1,3)$-Beta-D-Glucan Detection Reagent Kit 
(Associates of Cape Cod, East Falmouth, MA, USA), as per the manufacturer's directions. Briefly, $50 \mu \mathrm{l}$ of each sample were mixed with $50 \mu \mathrm{l}$ of Glucatell reagent. After incubation for $40 \mathrm{~min}$ at $37^{\circ} \mathrm{C}$, the reaction was stopped by adding sequentially $50 \mu \mathrm{l}$ of sodium nitrite, $50 \mu \mathrm{l}$ of ammonium sulphamate and then $50 \mu \mathrm{l}$ of $\mathrm{N}$-(1-napthyl) ethylenediamine dihydrochloride (NEDA). All solutions and glucan standards were supplied with the kit. The solution obtained was read in a microtitre plate reader (BioTek Synergy HT, Izasa, Portugal) at $540 \mathrm{~nm}$, using PBS as a blank. The values were normalized per $g$ of dry weight of biofilm and presented as ng of $\beta$-1,3-glucans per $g$ of dry weight of biofilm (ng g ${ }^{-1}$ biofilm).

\section{Ergosterol extraction and quantification}

For the ergosterol extraction, $2 \mathrm{ml}$ of $\mathrm{n}$-hexan (Thermo Scientific, Waltham, MA, USA) were added to $10 \mathrm{ml}$ of the matrix suspension prepared as previously described. This preparation was then submitted to vortex for $1 \mathrm{~min}$. This procedure was performed three times and the top solution sequestered to a $10 \mathrm{ml}$ amber bottle. After the extraction, the solutions were dried with nitrogen until all the organic solvent has evaporated. The dried extract was resuspended in $2 \mathrm{ml}$ of methanol, filtered with a $0.45 \mu \mathrm{m}$ filter into an Eppendorf tube and stored at $-20^{\circ} \mathrm{C}$ (Marín et al. 2006). For the ergosterol quantification, the high-pressure liquid chromatography (HPLC) method was performed in a Varian STAR 9002 (Varian, Walnut Creek, CA, USA) using a C18 column (YMC, Allentown, PA, USA). An isocratic mobile phase of $100 \%$ of methanol with a flow of $1 \mathrm{ml} \mathrm{min}{ }^{-1}$, for $20 \mathrm{~min}$, was used for the quantification of each sample. The results were automatically shown by the HPLC detector (Marín et al. 2006), and then normalized by $g$ of dry weight biofilm and presented as $\mu$ g of ergosterol per $\mathrm{g}$ of dry weight of biofilm ( $\mu \mathrm{g} \mathrm{g}^{-1}$ biofilm).

\section{Statistical analysis}

Data were analysed using Graph Pad Prism (v.7). Statistical significance was determined by one-way or two-way ANOVA with Tukey's multiple comparison post-test. All tests were performed with a confidence level of $95 \%$.

\section{Results}

\section{Characterization of C. albicans biofilms in the presence of lactic acid}

Lactic acid, naturally present in several sites in the human body, can be used as an alternative carbon source by Candida cells. To determine the influence of lactic acid on the formation and behaviour of C. albicans biofilms in vitro, they were characterized using C. albicans RM1000 (wild type) cells in RPMI-containing $0.5 \%$ lactic acid medium at $\mathrm{pH} 5$, a condition where most of the acid is present in its anionic form and its assimilation depends on a transporter-mediated system (Casal et al. 2008). In order to mimic different host microenvironments, and given that glucose is the preferential carbon source for C. albicans, assays using RPMI medium containing glucose, and no lactic acid, at both pH7 and pH5 were also carried out. Biofilms were analysed by total biomass quantification through CV staining and the enumeration of cultivable cells (CFUs) (Figure 1A and C). Both quantifications were carried out on biofilms grown for $48 \mathrm{~h}$. The ultrastructure of biofilms was assessed by SEM analysis (Figure $1 \mathrm{~B}$ and D).

The results obtained indicate that $C$. albicans WT cells displayed enhanced biofilm formation in the presence of lactate when compared to biofilms formed in the presence of glucose ( $p<0.001$; Figure 1A). It was also observed that, depending on $\mathrm{pH}$ and the carbon source, biofilms formed in the presence of lactate developed different structures and the cells exhibited different morphological characteristics. Mature biofilms grown in the presence of glucose at pH7 (RPMI pH7) consisted of a dense network of hyphae without yeast forms, while at pH5 (RPMI pH5) a reduction in filaments was evident (Figure 1B). In the presence of lactic acid (RPMI $0.5 \%$ lactic acid pH5) most of the cells were in the yeast form (Figure 1B). Both WT and mutant strains displayed an identical growth profile, exhibiting more biofilm biomass in the presence of lactic acid than in glucose $(p<0.001$; Figure 1A and C). Regarding ultrastructural and morphological features, the jen 1jen 2 double mutant mainly formed hyphae in the presence of glucose; while in the presence of lactic acid the yeast form was the most predominant with almost no filamentous forms observed (Figure 1D). The mutant strain also exhibited a compact biofilm structure with some cracks and less hyphae than the WT (Figure 1B and D).

\section{Lactic acid increases the susceptibility of C. albicans planktonic cells to fluconazole}

In an attempt to further explore the role of Jen1 and Jen2 in C. albicans resistance to fluconazole, the minimal fungicidal concentration (MFC) and the MIC by CFU counting for WT and jen1jen 2 planktonic cells grown either in lactic acid or glucose was determined (Table 1).

In RPMI medium, independently of the $\mathrm{pH}$, no differences in MIC values were observed between the tested strains, which displayed equal growth behaviour up to the highest concentration of fluconazole (Table 1). In the presence of lactic acid, a MIC value of $150 \mu \mathrm{g} \mathrm{ml}^{-1}$ and a MFC value of $312.5 \mu \mathrm{g} \mathrm{ml}^{-1}$ were obtained for the WT strain, and lower fluconazole MIC and MFC values (50 
(A)

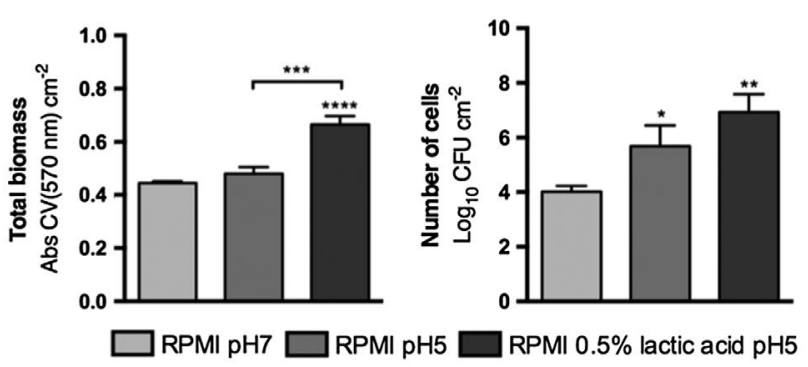

(B)
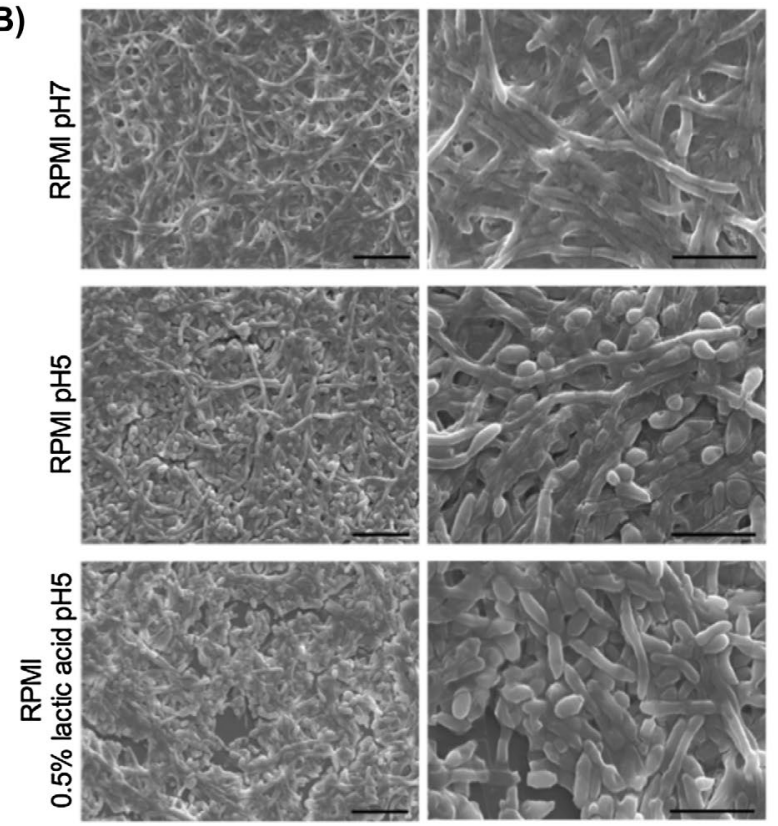

(C) jen1jen2 mutant strain

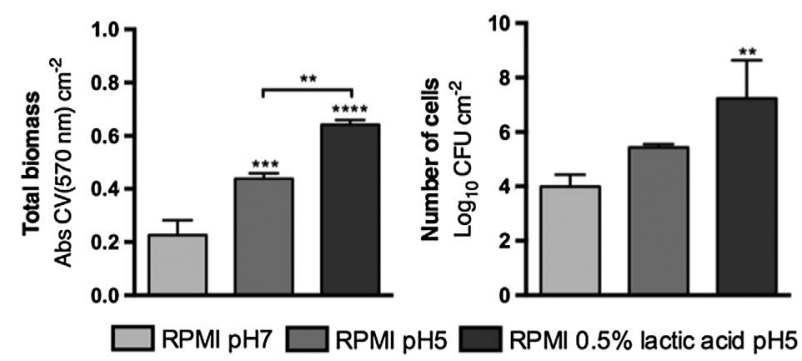

(D)
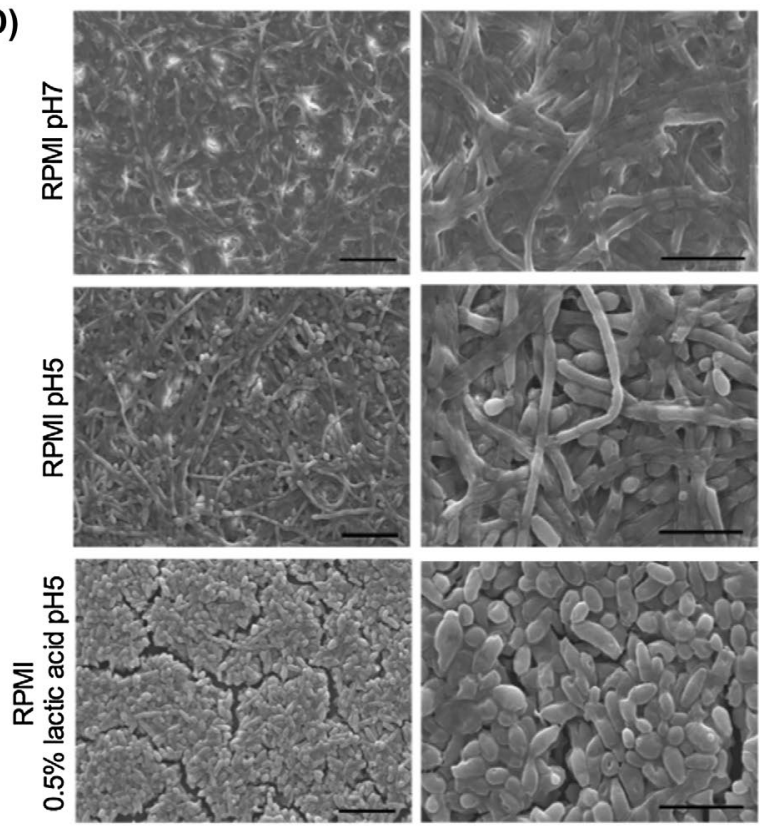

Figure 1. Effect of lactic acid on C. albicans biofilms (wild type strain vs the jen1jen2 mutant strain. (A, C) Total biomass of biofilms and number of cells in biofilms formed after $48 \mathrm{~h}$ in different conditions: RPMI pH7, RPMI pH5 and RPMI 0.5\% lactic acid pH5. The absorbance value of CV solution (Abs CV) at $570 \mathrm{~nm}$ and the logarithm of colony forming units (CFU) were normalized by unit of area (Abs $\mathrm{CV}_{570 \mathrm{~nm}} \mathrm{~cm}^{-2}$ and $\log _{10} \mathrm{CFU} \mathrm{cm}{ }^{-2}$, respectively). Error bars represent SDs. Statistically significant changes appear above error bars when compared with the control group (RPMI pH7) or above brackets when compared with the second control group (RPMI $\mathrm{pH} 5)$; ${ }^{* *}(p<0.01)$, ${ }^{* * *}(p<0.001)$. (B, D) SEM images of mature biofilms. The left and the right images in each panel represent magnifications of $1,000 \times$ and $3,000 \times$, respectively. Bars on the images correspond to $20 \mu \mathrm{m}$ at $1,000 \times$ magnification and $10 \mu \mathrm{m}$ at $3,000 \times$ magnification.

Table 1. Effect of fluconazole on C. albicans WT and jen1jen2 planktonic cells using different growth conditions: cells grown in RPMI pH7, RPMI pH5 and RPMI 0.5\% lactic acid pH5.

\begin{tabular}{llcc}
\hline Strain & \multicolumn{1}{c}{ Condition } & MIC $\left(\mu \mathrm{g} \mathrm{ml}^{-1}\right)$ & $\mathrm{MFC}\left(\mu \mathrm{g} \mathrm{ml}^{-1}\right)$ \\
\hline WT & RPMl pH7 & $\geq 1,250$ & $>1,250$ \\
& RPMl pH5 & 625 & 1,250 \\
& RPMI 0.5\% lactic acid pH5 & 150 & 312.5 \\
jen1jen2 & RPMl pH7 & $\geq 1,250$ & $>1,250$ \\
& RPMl pH5 & 625 & 1,250 \\
& RPMl 0.5\% lactic acid pH5 & 50 & 150 \\
\hline
\end{tabular}

Note: MICs were determined visually and MFCs by total number of CFUs, according to the microdilution method (CLSI 2008).

and $150 \mu \mathrm{g} \mathrm{ml}^{-1}$, respectively) were found for the mutant strain (Table 1). Therefore, with the addition of fluconazole, all strains became more susceptible in the presence of lactic acid and the double mutant was even more susceptible than the WT. These results suggest that Jen1 and Jen2 play a role in antifungal resistance to fluconazole.

\section{Characterization of fluconazole antifungal activity in C. albicans biofilms}

The effect of fluconazole was also evaluated for biofilms (Figure 2), similar to what was done for planktonic cells.

Figure $2 \mathrm{~A}$ shows that in RPMI medium at $\mathrm{pH} 7$, the presence of fluconazole did not significantly affect the amount of total biomass. Interestingly, at $\mathrm{pH} 5$ and in the presence of lactic acid, the reduction was significant for both strains. While in RPMI lactic acid jen1jen2 planktonic cells were more susceptible to fluconazole compared to the WT strain (Table 1), their biofilm counterparts did not follow the same behaviour. In general, a significant 
Table 2. Effect of fluconazole on ergosterol production in the biofilm matrices of C. albicans WT and the mutant strain jen1jen2.

\begin{tabular}{lccc}
\hline Strain & Condition & $\begin{array}{c}\text { Fluconazole } \\
\left(\mu \mathrm{g} \mathrm{ml}^{-1}\right)\end{array}$ & $\begin{array}{c}\text { Ergosterol } \\
\left(\mu \mathrm{g} \mathrm{g}^{-1} \text { biofilm }\right)\end{array}$ \\
\hline WT & RPMl pH7 & 0 & $\leq 14.360$ \\
& & 312.5 & $\leq 256.000$ \\
& RPMI pH5 & 1,250 & $\leq 33.750$ \\
& & 0 & $\leq 17.724$ \\
& & 312.5 & $\leq 27.442$ \\
& RPMI 0.5\% lactic & 1,250 & $\leq 9.333$ \\
jen1jen2 & 0 & $\leq 80.350$ \\
& acid pH5 & 312.5 & $\leq 105.560$ \\
& RPMI pH7 & 1,250 & $\leq 131.200$ \\
& & 0 & $\leq 30.000$ \\
& & 312.5 & $\leq 128.570$ \\
& RPMI pH5 & 1,250 & $\leq 23.680$ \\
& & 0 & $\leq 31.163$ \\
& & 312.5 & $\leq 11.224$ \\
& RPMI 0.5\% lactic & 1,250 & $\leq 9.063$ \\
& acid pH5 & 0 & $\leq 60.000$ \\
& & 312.5 & $\leq 68.870$ \\
& & 1,250 & $\leq 0.220$ \\
\hline
\end{tabular}

Note: The table displays the highest concentrations detected in $\mu \mathrm{g}$ per $\mathrm{g}$ of $48 \mathrm{~h}$ biofilm dry weight ( $\mu \mathrm{g} \mathrm{g}^{-1}$ biofilm).

reduction in cell viability was only observed at the highest tested concentration of fluconazole (Figure 2A). The effect of fluconazole on biofilm structure was also assessed by SEM analysis (Figure 2B). In RPMI medium, the differences were only visible with $1,250 \mu \mathrm{g} \mathrm{ml}^{-1}$ of fluconazole for both strains and conditions. In the case of RPMI lactic acid, significant differences were observed between both strains in the presence and in the absence of fluconazole (Figure 2B). The absence of an antifungal resulted in a dense, compact biofilm with almost no hyphal forms in the mutant strain, in contrast to a hyphae containing biofilm structure in the WT strain. Interestingly, the highest fluconazole concentration led to an increase in hyphae formation both for WT and mutant strains (Figure 2B).

\section{Impact of carbon sources and fluconazole treatment on the biofilm matrix of $C$. albicans}

The matrix of Candida has a prominent role in the antifungal drug resistance associated with biofilms (Branda et al. 2005; Zarnowski et al. 2014). The biofilm matrix composition of both strains was analysed in response to changes in carbon sources and antifungal treatment. Several components were evaluated that have been involved in the sequestration of antifungals, such as polysaccharides (Figure 3A), the extracellular carbohydrate $\beta$-1,3-glucan (Figure 3B), proteins (Figure 3C) and ergosterol (Table 2).

The content of polysaccharides increased in the presence of fluconazole, particularly in the highest concentration, in all tested conditions (Figure 3A). However, in RPMI pH5 medium this increase was lower in both strains. A significant decrease in polysaccharides in the jen1jen 2 mutant strain was observed, when compared with the $\mathrm{WT}$, in the presence of lactate and high concentrations of fluconazole (Figure $3 \mathrm{~A}$ ). This result suggests that there may be a greater requirement for Jen $1 / 2$-mediated lactate uptake, in these stress conditions.

Inversely, the amount of $\beta$-1,3-glucans found in the biofilm matrices did not follow the same pattern in the growth conditions tested, in response to antifungal treatment (Figure 3B). The total protein content, in contrast to what was observed for the polysaccharides, decreased in the presence of fluconazole, in both strains (Figure 3C).

In general, the concentration of ergosterol in the matrices increased with the addition of fluconazole up to $312.5 \mu \mathrm{g} \mathrm{ml}^{-1}$ (Table 2). However, the addition of $1,250 \mu \mathrm{g} \mathrm{ml}^{-1}$ of fluconazole inverted this tendency, with the exception of matrices extracted from WT biofilms, grown in the presence of lactic acid. As observed for the polysaccharides, the jen1jen 2 mutant strain displayed a lower amount of ergosterol when compared with WT, in the presence of lactate and $1,250 \mu \mathrm{g} \mathrm{ml}^{-1}$ of fluconazole.

\section{Discussion}

Candida biofilms can occur on most biological and non-biologic substrata, such as catheters, serving as reservoirs or sources for potential infections (Donlan and Costerton 2002; Douglas 2003; Ramage et al. 2009). Most studies of Candida species, including Candida biofilms, have been carried out using glucose as the sole carbon source in the medium. However, the concentration of glucose in some host niches is usually limited and Candida depends on alternative substrates for growth and invasion. The physiology of Candida is profoundly affected by carbon sources so it is crucial to accurately reflect this variable in experiments in order to understand biofilm formation in these niches. Some studies have shown that lactate-grown cells, in minimal medium, adhere more strongly to plastic surfaces and display increased biofilm formation (Ene et al. 2012, 2013). However, the growth conditions used in this study did not promote yeast hyphae transitions and classical biofilm formation (Ene et al. 2012). In this context, C. albicans biofilm formation was studied in media containing both glucose and lactate at different $\mathrm{pH}$ values, conditions that mimic host microenvironments where this pathogen can be found.

The presence of lactic acid promoted biofilm formation (Figure 1A). Regarding morphology, C. albicans biofilms can comprise yeast cells, hyphae and pseudohyphae (Ramage et al. 2005). It is well established that $C$. albicans changes its morphology according to environmental conditions, which constitutes one of its virulence factors (Calderone and Fonzi 2001). Nutrient starvation, the presence of serum, an increase in temperature and $\mathrm{pH}$ have been reported as hypha-inducing environmental 
(A) RPMI pH7
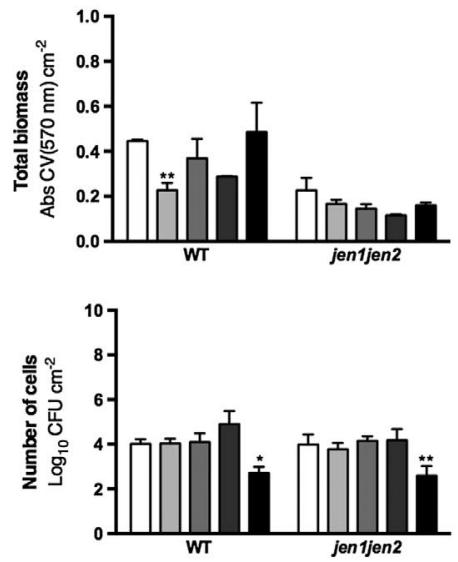

RPMI pH5
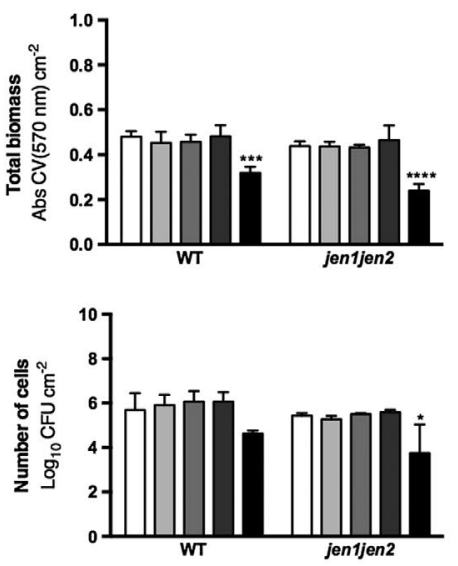

RPMI $0.5 \%$ lactic acid pH5
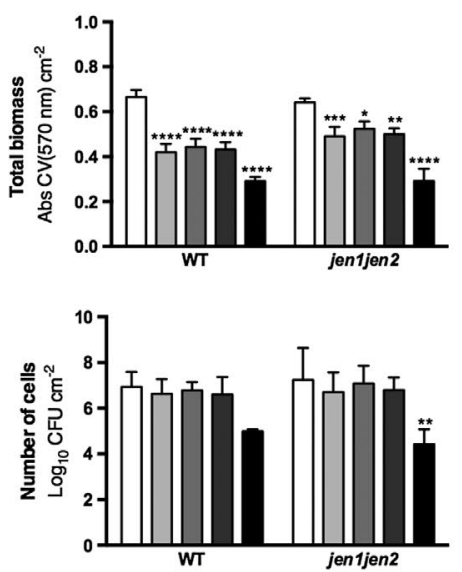

$\square 0 \mu \mathrm{g} \mathrm{ml}^{-1} \square 50 \mathrm{~g} \mathrm{ml}^{-1} \square 150 \mu \mathrm{g} \mathrm{ml}^{-1} \square 312,5 \mathrm{gg} \mathrm{ml}^{-1} \square 1250 \mu \mathrm{g} \mathrm{ml}^{-1}$

(B)

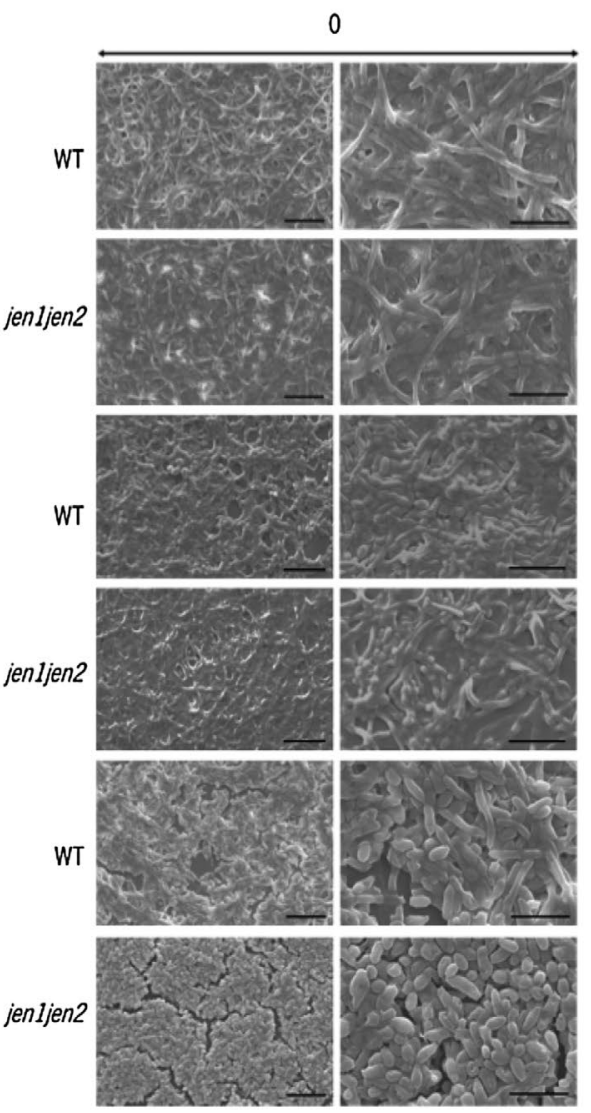

[Fluconazole] $\mu \mathrm{g} \mathrm{ml}^{-1}$

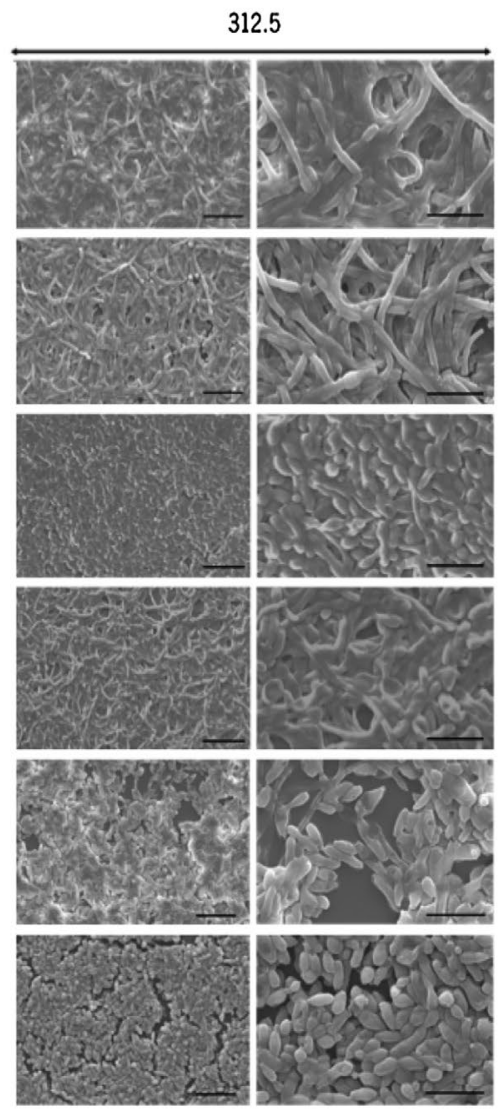

송
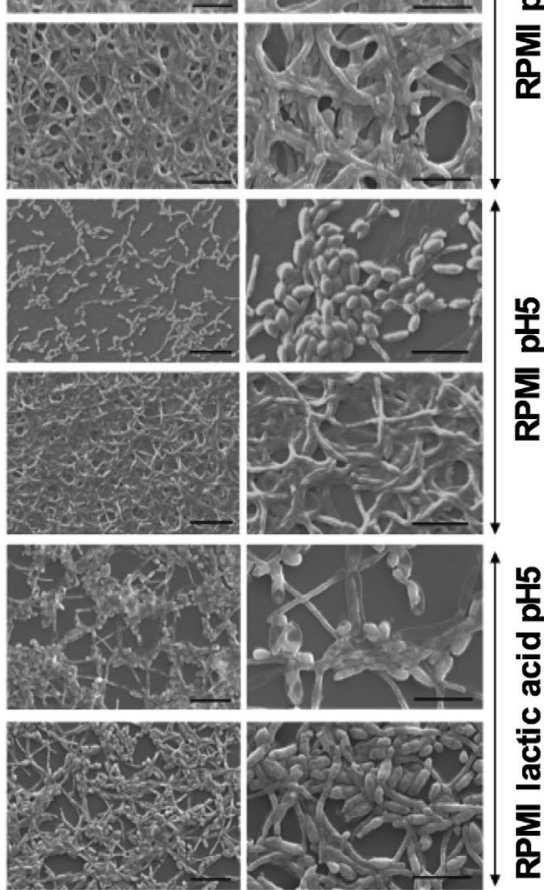

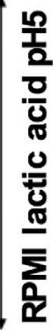

Figure 2. Effect of fluconazole on C. albicans WT and jen1jen2 biofilms grown in RPMI pH7, RPMI pH5 and RPMI $0.5 \%$ lactic acid pH5. (A) Histograms at the top represent the absorbance values of CV solution at $570 \mathrm{~nm}$ normalized by unit of area $\left(\mathrm{Abs}_{\mathrm{CV}} \mathrm{CV}_{570 \mathrm{~nm}} \mathrm{Cm}^{-2}\right)$ and histograms at the bottom represent the logarithm of CFUs normalized by unit of area $\left(\log _{10} \mathrm{CFU} \mathrm{cm}^{-2}\right)$ of biofilm. The legend displays the different fluconazole concentrations used. Error bars represent SDs. ${ }^{*},{ }^{* *},{ }^{* * *}$ and ${ }^{* * * *}$ mean that results are statistically significant ( $p<0.05, p<0.01, p<0.001$ and $p<0.0001$, respectively). (B) SEM images showing the effect of fluconazole on both WT and jen1jen 2 biofilms. The left and the right image in each strain for the respective fluconazole concentration represents a magnification of $1,000 \times$ and 3,000 $\times$, respectively. Bars in the images correspond to $20 \mu \mathrm{m}$ at 1,000 $\times$ magnification and $10 \mu \mathrm{m}$ at 3,000 $\times$ magnification. 


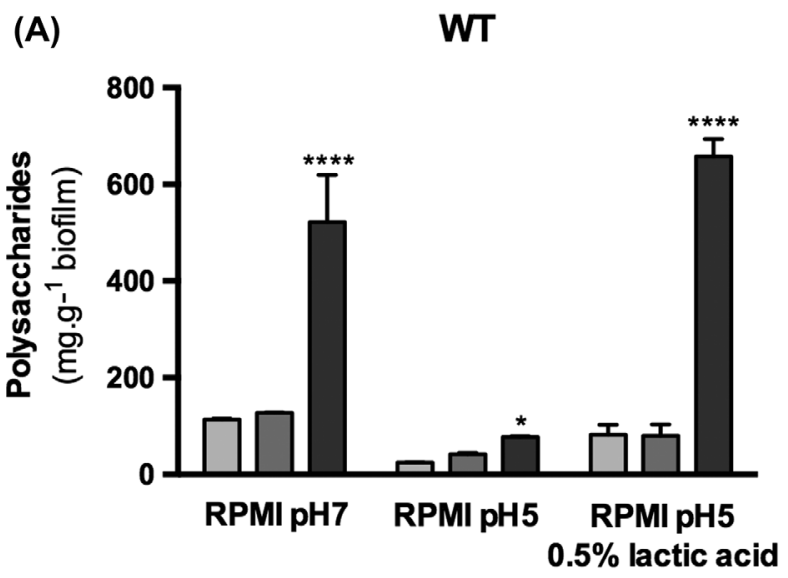

(B)

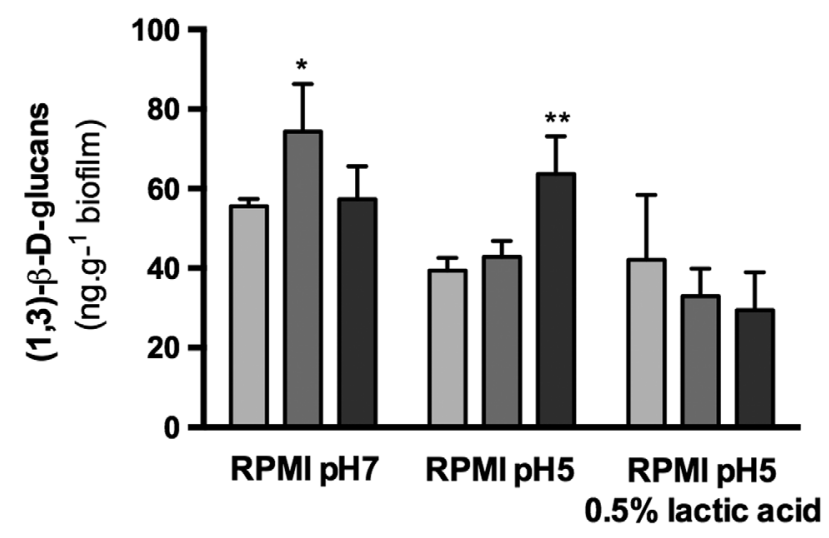

(C)

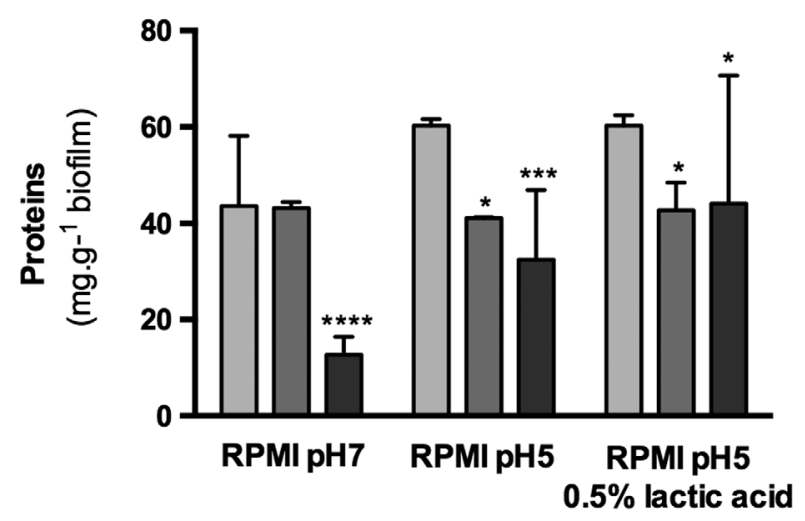

jen1jen2

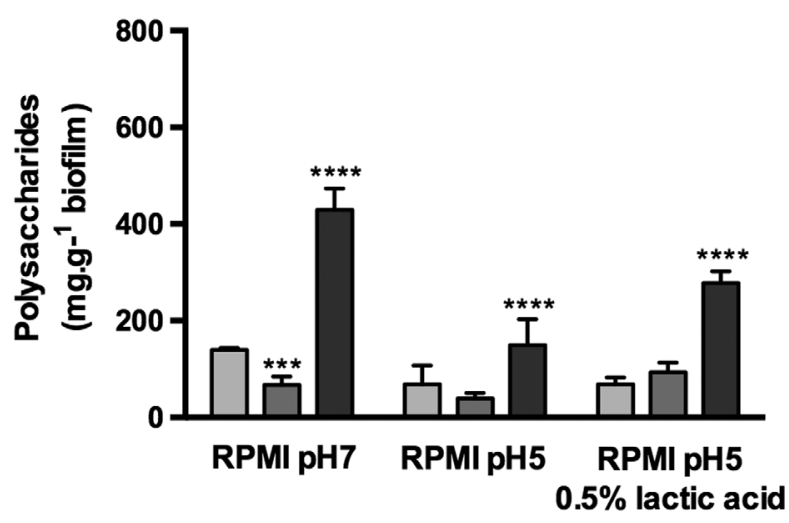

jen1jen2

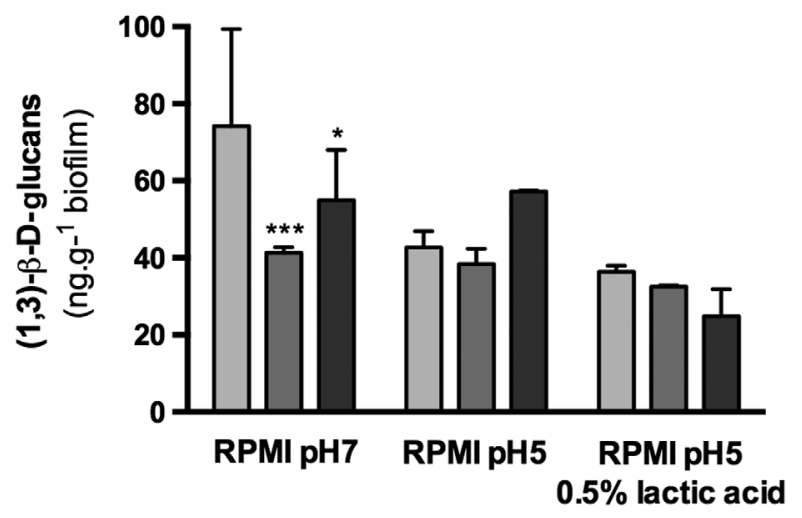

jen1jen2

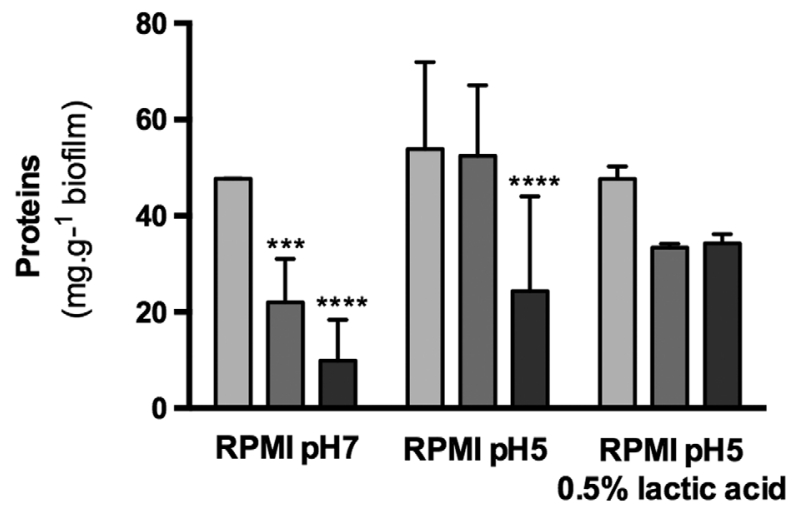

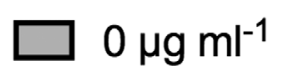

$\square 312.5 \mu \mathrm{g} \mathrm{ml}^{-1}$

$\square 1250 \mu \mathrm{g} \mathrm{ml}^{-1}$

Figure 3. Effect of fluconazole on the biofilm matrix composition of C. albicans WT and jen1jen2 strains grown in RPMI pH7, RPMI pH5 and RPMI $0.5 \%$ lactic acid pH5. Concentrations are presented in $\mathrm{mg}$ for (A) polysaccharides and (C) proteins or $\mathrm{ng}$ for (B) 1,3- $\beta$-D-glucans per $\mathrm{g}$ of $48 \mathrm{~h}$ biofilm dry weight ( $\mathrm{mg} \mathrm{g}^{-1}$ biofilm or $\mathrm{ng} \mathrm{g}^{-1}$ biofilm, respectively). Error bars represent SDs. ${ }^{*},{ }^{* *},{ }^{* * *}$ and ${ }^{* * * *}$ mean that the results are statistically significant $(p<0.05, p<0.01, p<0.001$ and $p<0.0001$, respectively).

factors (Whiteway and Bachewich 2007). As expected, the present study shows that in RPMI pH7 the hyphal morphology predominated, whereas in RPMI pH5 the yeast form was the most prevalent (Figure 1B). However, in the presence of lactate (RPMI $0.5 \%$ lactic acid pH5) the yeast form was even more predominant and minimal hyphal formation was observed (Figure 1B). In addition to $\mathrm{pH}$, the carbon source available is also important in $C$. 
albicans morphology and ultimately in C. albicans biofilm formation.

Regarding the contribution of the carboxylic acid transporters Jen 1 and Jen2, the disruption of JEN1 and JEN2 compromises biofilm formation in RPMI containing lactate. These results indicate that the jenljen 2 mutant is capable of forming biofilm in all the tested conditions and its behaviour is similar to that of WT, as both strains have more biofilm biomass in the presence of lactic acid (Figure 1A and C). Regarding ultrastructure, the jen1jen 2 mutant presented a compact biofilm structure with some cracks and even less hyphae than the WT in lactate containing media (Figure 1D). These differences, observed in the presence of lactate, point to an involvement of these transporters in biofilm formation, at least in terms of ultrastructure.

Biofilm cells display unique phenotypic characteristics, the most remarkable of which is that they are particularly resistant to both antifungal agents and host immune factors (Douglas 2003; Ramage et al. 2009). Attempts were also made to elucidate the role of Jen 1 and Jen2 in $C$. albicans resistance to the commonly used antifungal drug fluconazole. In a jen1jen 2 double mutant strain, carboxylic acids only enter the cell by simple diffusion of the undissociated form of the acid, a process dependent on extracellular $\mathrm{pH}$. These studies were conducted both in planktonic and biofilm cells. The results indicate that lactate-grown planktonic cells of the mutant strain were more susceptible to fluconazole than the WT cells (Table 1), pointing to an involvement of these transporters in antifungal drug resistance. Additionally, the presence of lactic acid in the medium acts synergistically with fluconazole, since WT cells became more susceptible to fluconazole under this condition. These results are in agreement with previous studies, that showed an increase in C. albicans susceptibility to miconazole, another azole antifungal drug, in lactic acid-grown cells (Ene et al. 2012), and to fluconazole in a synthetic vagina-simulative medium in an acidic pH (Moosa et al. 2004). The analysis of the components of the medium indicated that acetic acid was responsible for the synergistic, fungicidal effect and not lactic acid (Moosa et al. 2004). Here, the results indicate that lactic acid also triggers this effect and maybe, in this niche, both acids can contribute equally to this phenomenon. These findings support the results from Ene et al. (2012) with the demonstration of growth inhibition by a clinically important azole, reinforcing the idea that lactate reduces azole resistance. Regarding biofilms, fluconazole had almost no effect on biofilm reduction (Table 1). While in the presence of lactic acid jenljen2 planktonic cells were more susceptible to fluconazole compared to WT cells, their biofilm counterparts did not follow the same behaviour. In general, a significant reduction in cell viability was only observed at the highest tested concentration of fluconazole (Table 2). However, these results should be interpreted carefully since in planktonic cells, according to the standard method used (CLSI 2008), the antifungal drug is added at the beginning of the assay, and in biofilms the drug is added after $24 \mathrm{~h}$. These different procedures, inherent in biofilm growth assays, may be responsible for the observed phenotypes. It was reported that the addition of fluconazole at the initiation of biofilm formation inhibited this process even in C. albicans strains known to be resistant to fluconazole (Bruzual et al. 2007). Nevertheless, these results are in accordance with what has been reported in other studies that demonstrated that biofilms need about 100 to 1,000 times more of an antifungal drug to be eradicated (Baillie and Douglas 1998; Chandra et al. 2001; Ramage et al. 2001). An interesting phenotype was observed as a result of fluconazole treatment in the RPMI medium containing lactic acid. In this condition both strains showed increased hyphae formation (Figure 2B), an important feature of this fungus in pathogenesis. According to previous studies, hypha formation in planktonic cells of C. albicans is inhibited in the presence of fluconazole or other azole derivatives (Odds and Kerridge 1985; Ha and White 1999). However, in the case of biofilms, the cells are immersed in an extracellular matrix that provides better protection against stress factors.

Antifungal resistance in biofilms is complex. It can be inducible in response to a drug, or an irreversible genetic change resulting from a prolonged exposure. The mechanisms utilized by fungi to combat the effects of antifungal drugs comprise modifications or overexpression of target molecules, active extrusion through efflux pumps, limited diffusion, tolerance, and cell density (Donlan and Costerton 2002; Kumamoto 2002; Tobudic et al. 2012). Recent studies suggest that the composition of the extracellular matrix and its regulation might play a central role in resistance, with the polysaccharide $\beta$-1,3-glucan being linked to biofilm protection against antifungal agents (Nett et al. 2007b, 2010; Taff et al. 2012). Besides conferring significant resistance to antifungal therapy, the composition of the extracellular matrix also contributes to defence from the host immune response and maintenance of nutrients (Cuellar-Cruz et al. 2012). The matrix contains polysaccharides, small amounts of proteins, DNA and can also have several other components (Douglas 2003; Nett and Andes 2006; Martins et al. 2010; CuellarCruz et al. 2012). The results of this study strengthen the role of polysaccharides in antifungal drug resistance. The increase in this component upon fluconazole treatment could reflect a cell mechanism for survival under the presence of the drug. This reinforces the resistance model which suggests that the polysaccharides present in the extracellular matrix that covers biofilm cells are 
capable of sequestering antifungal drugs, acting as a 'drug sponge. This has the ability to prevent invading cells from reaching their targets, thus limiting their toxicity to these cells and associated transcriptional responses (Nett et al. 2007a, 2010; Taff et al. 2012). Additionally, these results also show that in the presence of lactic acid and at high concentrations of fluconazole, the production of general polysaccharides is affected in a jen1jen 2 mutant strain (Figure 3A). This suggests that Jen $1 / 2$-mediated lactate uptake or Jen $1 / 2$-mediated signalling is required in these conditions. However, the amount of $\beta$-1,3-glucans found in the biofilm matrices was almost constant in all conditions (Figure 3B), suggesting that these molecules only represent a small portion of the total matrix polysaccharides (Zarnowski et al. 2014). In contrast with the polysaccharides, the matrices from all strains and conditions exhibited a decrease in the protein content with the antifungal treatment (Figure 3C), suggesting a possible compensatory mechanism in the matrix content. Another mechanism of resistance is the overexpression of target molecules. It has been reported that the exposure of biofilms to fluconazole results in overexpression of the genes encoding the enzymes involved in ergosterol biosynthesis (Nailis et al. 2010). An increase in ergosterol in the extracellular matrix was observed with fluconazole treatment (up to $312.5 \mu \mathrm{g} \mathrm{ml}^{-1}$ ) and a decrease in the presence of the highest concentration of the drug (Table 2). This may be due to differential regulation of ergosterol genes associated with antifungal treatment.

In summary, this work indicates that the alternative carbon source lactic acid affects the structure and morphology of C. albicans biofilms and has an impact on antifungal resistance to fluconazole in both planktonic and biofilm cells. It also suggests that the carboxylic acid transporters Jen 1 and Jen 2 might play a role in these processes during growth in host microenvironments containing lactate. These findings have an important clinical impact, namely in the treatment of vaginal candidiasis, a host niche with an acidic $\mathrm{pH}$, rich in alternative carbon sources, such as lactate.

\section{Acknowledgements}

The authors would like to acknowledge Pfizer, S.A. for the kind donation of fluconazole.

\section{Disclosure statement}

No potential conflict of interest was reported by the authors.

\section{Funding}

This study was funded by the Portuguese Foundation for Science and Technology (FCT) [grant number PTDC/BIAM-
IC/5184/2014]. SM, CFR and RA received FCT PhD studentships [grant numbers SFRH/BD/74790/2010, SFRH/ $\mathrm{BD} / 93078 / 2013$, PD/BD/113813/2015, respectively]. The work on CBMA was supported by FCT [grant number UID/ BIA/04050/2013] and COMPETE 2020 [grant number POCI01-0145-FEDER-007569]. The work on CEB was supported by FCT [grant number UID/BIO/04469/2013], COMPETE 2020 [grant number POCI-01-0145-FEDER-006684] and BioTecNorte operation [grant number NORTE-01-0145FEDER-000004] funded by the ERDF under the scope of Norte2020 - Programa Operacional Regional do Norte. AJPB was funded by the UK Medical Research Council [grant number MR/M026663/1]; by the UK Biotechnology and Biological Research Council [grant number BB/K017365/1]; and by the MRC Centre for Medical Mycology and the University of Aberdeen [grant number MR/M026663/1]. The funders had no role in study design, data collection and analysis, the decision to publish, or in the preparation of the manuscript.

\section{References}

Baillie GS, Douglas LJ. 1998. Iron-limited biofilms of Candida albicans and their susceptibility to amphotericin B. Antimicrob Agents Chemother. 42:2146-2149.

Ballou ER, Avelar GM, Childers DS, Mackie J, Bain JM, Wagener J, Kastora SL, Panea MD, Hardison SE, Walker LA, et al. 2016. Lactate signalling regulates fungal $\beta$-glucan masking and immune evasion. Nat Microbiol. 2:16238.

Barelle CJ, Priest CL, MacCallum DM, Gow NAR, Odds FC, Brown AJP. 2006. Niche-specific regulation of central metabolic pathways in a fungal pathogen. Cell Microbiol. 8:961-971.

Branda SS, Vik A, Friedman L, Kolter R. 2005. Biofilms: the matrix revisited. Trends Microbiol. 13:20-26.

Brown AJP, Brown GD, Netea MG, Gow NAR. 2014. Metabolism impacts upon Candida immunogenicity and pathogenicity at multiple levels. Trends Microbiol. 22:614622.

Bruzual I, Riggle P, Hadley S, Kumamoto CA. 2007. Biofilm formation by fluconazole-resistant Candida albicans strains is inhibited by fluconazole. J Antimicrob Chemother. 59:441-450.

Calderone R, Fonzi W. 2001. Virulence factors of Candida albicans. Trends Microbiol. 9:327-335.

Casal M, Paiva S, Queirós O, Soares-Silva I. 2008. Transport of carboxylic acids in yeasts. FEMS Microbiol Rev. 32:974-994.

Chandra J, Kuhn DM, Mukherjee PK, Hoyer LL, McCormick T, Ghannoum MA. 2001. Biofilm formation by the fungal pathogen Candida albicans: development, architecture, and drug resistance. J Bacteriol. 183:5385-5394.

CLSI. 2008. Clinical and Laboratory Standards Institute: reference method for broth dilution antifungal susceptibility testing of yeasts; approved standard-; CLSI document M27-A3.

Cuellar-Cruz M, Lopez-Romero E, Villagomez-Castro JC, Ruiz-Baca E. 2012. Candida species: new insights into biofilm formation. Future Microbiol. 7:755-771.

Davis DA. 2009. How human pathogenic fungi sense and adapt to $\mathrm{pH}$ : the link to virulence. Curr Opin Microbiol. 12:365370.

De Bernardis F, Mühlschlegel FA, Cassone A, Fonzi WA. 1998. The $\mathrm{pH}$ of the host niche controls gene expression in and virulence of Candida albicans. Infect Immun. 66:3317-3325. 
Donelli G, Vuotto C. 2014. Biofilm-based infections in longterm care facilities. Future Microbiol. 9:175-188.

Donlan RM, Costerton JW. 2002. Biofilms: survival mechanisms of clinically relevant microorganisms. Clin Microbiol Rev. 15:167-193.

Douglas LJ. 2003. Candida biofilms and their role in infection. Trends Microbiol. 11:30-36.

DuBois M, Gilles KA, Hamilton JK, Rebers PA, Smith F. 1956. Colorimetric method for determination of sugars and related substances. Anal Chem. 28:350-356.

Ene IV, Adya AK, Wehmeier S, Brand AC, MacCallum DM, Gow NAR, Brown AJP. 2012. Host carbon sources modulate cell wall architecture, drug resistance and virulence in a fungal pathogen. Cell Microbiol. 14:1319-1335.

Ene IV, Cheng SC, Netea MG, Brown AJP. 2013. Growth of Candida albicans cells on the physiologically relevant carbon source lactate affects their recognition and phagocytosis by immune cells. Infect Immun. 81:238-248.

Flint HJ, Scott KP, Louis P, Duncan SH. 2012. The role of the gut microbiota in nutrition and health. Nat Rev Gastroenterol Hepatol. 9:577-589.

Ha KC, White TC. 1999. Effects of azole antifungal drugs on the transition from yeast cells to hyphae in susceptible and resistant isolates of the pathogenic yeast Candida albicans. Antimicrob Agents Chemother. 43:763-768.

Kumamoto CA. 2002. Candida biofilms. Curr Opin Microbiol. 5:608-611.

Lorenz MC, Bender JA, Fink GR. 2004. Transcriptional response of Candida albicans upon internalization by macrophages. Eukaryot Cell. 3:1076-1087.

Lorenz MC, Fink GR. 2001. The glyoxylate cycle is required for fungal virulence. Nature. 412:83-86.

Marín S, Morales H, Ramos AJ, Sanchis V. 2006. Evaluation of growth quantification methods for modelling the growth of Penicillium expansum in an apple-based medium. J Sci Food Agric. 86:1468-1474.

Martins M, Uppuluri P, Thomas DP, Cleary IA, Henriques M, Lopez-Ribot JL, Oliveira R. 2010. Presence of extracellular DNA in the Candida albicans biofilm matrix and its contribution to biofilms. Mycopathologia. 169:323-331.

Miramón P, Dunker C, Windecker H, Bohovych IM, Brown AJP, Kurzai O, Hube B. 2012. Cellular responses of Candida albicans to phagocytosis and the extracellular activities of neutrophils are critical to counteract carbohydrate starvation, oxidative and nitrosative stress. PLOS ONE. 7.

Miramón P, Lorenz MC. 2017. A feast for Candida: metabolic plasticity confers an edge for virulence. PLoS Pathog. 13.

Moosa MYS, Sobel JD, Elhalis H, Du W, Akins RA. 2004. Fungicidal activity of fluconazole against Candida albicans in a synthetic vagina-simulative medium. Antimicrob Agents Chemother. 48:161-167.

Mota S, Alves R, Carneiro C, Silva S, Brown AJ, Istel F, Kuchler K, Sampaio P, Casal M, Henriques M, Paiva S. 2015. Candida glabrata susceptibility to antifungals and phagocytosis is modulated by acetate. Front Microbiol. 6 .

Nailis H, Vandenbosch D, Deforce D, Nelis HJ, Coenye T. 2010. Transcriptional response to fluconazole and amphotericin B in Candida albicans biofilms. Res Microbiol. 161:284-292.

Negredo A, Monteoliva L, Gil C, Pla J, Nombela C. 1997. Cloning, analysis and one-step disruption of the ARG5,6 gene of Candida albicans. Microbiology. 143:297-302.
Nett J, Andes D. 2006. Candida albicans biofilm development, modeling a host-pathogen interaction. Curr Opin Microbiol. 9:340-345.

Nett J, Lincoln L, Marchillo K, Andes D. 2007a. B-1,3 Glucan as a test for central venous catheter biofilm infection. J Infect Dis. 195:1705-1712.

Nett J, Lincoln L, Marchillo K, Massey R, Holoyda K, Hoff B, VanHandel M, Andes D. 2007b. Putative role of $\beta-1,3$ glucans in Candida albicans biofilm resistance. Antimicrob Agents Chemother. 51:510-520.

Nett JE, Sanchez H, Cain MT, Andes DR. 2010. Genetic basis of candida biofilm resistance due to drug-sequestering matrix glucan. J Infect Dis. 202:171-175.

Odds FC, Kerridge D. 1985. Morphogenesis in Candida albicans. CRC Crit Rev Microbiol. 12:45-93.

Pappas PG, Kauffman CA, Andes D, Benjamin DK Jr, Calandra TF, Edwards JE Jr, Filler SG, Fisher JF, Kullberg B, OstroskyZeichner L, et al. 2009. Clinical practice guidelines for the management of Candidiasis: 2009 update by the infectious diseases society of America. Clin Infect Dis [Internet]. 48:503-535.

Pfaller MA, Diekema DJ. 2007. Epidemiology of invasive Candidiasis: a persistent public health problem. Clin Microbiol Rev. 20:133-163.

Pfaller MA, Diekema DJ. 2010. Epidemiology of invasive mycoses in North America. Crit Rev Microbiol. 36:1-53.

Ramage G, Martínez JP, López-Ribot JL. 2006. Candida biofilms on implanted biomaterials: a clinically significant problem. FEMS Yeast Res. 6:979-986.

Ramage G, Mowat E, Jones B, Williams C, Lopez-Ribot J. 2009. Our current understanding of fungal biofilms. Crit Rev Microbiol. 35:340-355.

Ramage G, Saville SP, Thomas DP, López-Ribot JL. 2005. Candida biofilms: an update. Eukaryot Cell. 4:633-638.

Ramage G, Vandewalle K, Wickes BL, López-Ribot JL. 2001. Characteristics of biofilm formation by Candida albicans. Rev Iberoam Micol organo la Asoc Esp Espec en Micol. 18:163-170.

Silva S, Henriques M, Martins A, Oliveira R, Williams D, Azeredo J. 2009. Biofilms of non-Candida albicans Candida species: quantification, structure and matrix composition. Med Mycol. 47:681-689.

Soares-Silva I, Paiva S, Kötter P, Entian K-D, Casal M. 2004. The disruption of JEN1 from Candida albicans impairs the transport of lactate. Mol Membr Biol. 21:403-411.

Staib P, Kretschmar M, Nichterlein T, Kohler G, Michel S, Hof H, Hacker J, Morschhauser J. 1999. Host-induced, stage-specific virulence gene activation in Candida albicans during infection. Mol Microbiol. 32:533-546.

Stepanovic S, Vukovic D, Dakic I, Savic B, Švabić-Vlahović M. 2000. A modified microtiter-plate test for quantification of staphylococcal biofilm formation. J Microbiol Methods. 40:175-179.

Taff HT, Nett JE, Zarnowski R, Ross KM, Sanchez H, Cain MT, Hamaker J, Mitchell AP, Andes DR. 2012. A Candida biofilm-induced pathway for matrix glucan delivery: implications for drug resistance. PLoS Pathog. 8.

Tobudic S, Kratzer C, Presterl E. 2012. Azole-resistant Candida spp. - emerging pathogens? Mycoses. 55:24-32.

Vieira N, Casal M, Johansson B, MacCallum DM, Brown AJP, Paiva S. 2010. Functional specialization and differential 
regulation of short-chain carboxylic acid transporters in the pathogen Candida albicans. Mol Microbiol. 75:1337-1354.

Vylkova S, Carman AJ, Danhof HA, Collette JR, Zhou H, Lorenz MC. 2011. The fungal pathogen Candida albicans autoinduces hyphal morphogenesis by raising extracellular pH. MBio. 2.

Vylkova S, Lorenz MC. 2014. Modulation of phagosomal $\mathrm{pH}$ by Candida albicans promotes hyphal morphogenesis and requires Stp2p, a regulator of amino acid transport. PLoS Pathog. 10.

Whiteway M, Bachewich C. 2007. Morphogenesis in Candida albicans. Annu Rev Microbiol. 61:529-553.

Zarnowski R, Westler WM, de Lacmbouh GA, Marita JM, Bothe JR, Bernhardt J, Lounes-Hadj Sahraoui A, Fontaine J, Sanchez H, Hatfield RD, et al. 2014. Novel entries in a fungal biofilm matrix encyclopedia. MBio. 5:e01333-e01314. 